\title{
Theoretical investigation of the Thermophysical Properties of a R141b Based Nanorefrigerant-A Comparative Analysis
}

\author{
A.D. Parekh, V. Nair, P.R. Tailor \\ Department of Mechanical Engineering, SardarVallabhbhai National Institute of Technology, \\ Surat, Gujarat, 395007, India
}

\begin{abstract}
The Nanoparticle suspended refrigerants or nanorefrigerants have been under the researchers' scrutiny from past few years now. It is a well-established fact that the addition of nanoparticle into the refrigerant leads to better boiling heat performance which consequently leads to a better and more energy efficient refrigeration system. But, the knowledge of the thermophysical properties of the nanorefrigerants is still in its infancy. This theoretical investigation is an attempt to provide a new insight into the thermophysical properties such as thermal conductivity, thermal diffusivity, viscosity, specific heat and density of R141b based nanorefrigerants. The thermal conductivity enhancement using various models and mechanisms have been evaluated, compared and discussed in this work. This investigation was carried out on Cu-R141b, CuO-R141b, Al-R141b and $\mathrm{Al}_{2} \mathrm{O}_{3}-\mathrm{R} 141 \mathrm{~b}$ nanorefrigerant. The particle diameter was set at $50 \mathrm{~nm}$.
\end{abstract}

Keywords:Nanorefrigerants; Nanofluids; Thermophysical properties; Thermal conductivity; Viscosity; Thermal diffusivity

\section{Introduction}

Nanorefrigerants are homogenous mixtures of uniformly suspended nanoparticles in a base refrigerant. The intention behind dispersing nanoparticles into the refrigerant is to enhance the boiling heat transfer coefficient of the refrigerant which will eventually increase the overall heat transfer coefficient of the system. The refrigeration and air conditioning industry consumes $15 \%$ of the total electricity worldwide[1]. Consequently, it has become very important to work on more energy efficient refrigerants. Fortunately, there are many researchers and reviewers who have confirmed the improved refrigerant performance and system performance due to the addition of nanoparticles in the refrigerant [2-6].

The objective of this paper is to investigate the variation in thermophysical properties of various $\mathrm{R} 141 \mathrm{~b}$ based nanorefrigerants. R141b is chosen for this investigation because it is much easier to prepare R141b based nanorefrigerants and hence, it will be easier to validate the theoretical findings of this work through future experiments. As of now, the main focal point of the nanorefrigerant research is not the refrigerant itself .It is more important to analyse and understand the variation and the nature of variation of thermophysical properties of the nanorefrigerants independent of the refrigerant. Therefore, it is important to first establish the appropriate models and correlations of nanorefrigerants and then later on, these analyses can be extended to more conventional refrigerants. The thermophysical properties such as the thermal conductivity, density and specific heat will be investigated in this work. The highlight of this work is the study of thermal diffusivity of nanorefrigerants which was surprisingly ignored by many researchers in past. The study of thermal diffusivity revealed few startling results and gives a new insight into the field of nanorefrigerants.

\section{Methodology}

The nanorefrigerant thermophysical properties in this work are evaluated using various models and correlations available in open literature. This analysis is mainly focused on $\mathrm{Cu} / \mathrm{R} 141 \mathrm{~b}, \mathrm{CuO} / \mathrm{R} 141 \mathrm{~b}$, $\mathrm{Al} 2 \mathrm{O} 3 / \mathrm{R} 141 \mathrm{~b}$ and Al/R141b nanorefrigerants. The $\mathrm{CuO}$ and $\mathrm{Al} 2 \mathrm{O} 3$ nanoparticles are chosen mainly because of their excellent stability characteristics. The oxide nanoparticles are known to be more stable in nanofluid and nanorefrigerant solution [13]. But, the researchers must also consider working with metallic nanoparticles such 
as $\mathrm{Cu}$ and $\mathrm{Al}$ simply because these particles have superior thermal conductivity in comparison to their oxide counterparts. R141b has been chosen for this investigation because it is much easier to prepare R141b based nanorefrigerants. Hence, the results predicted in this work can be easily reproduced and verified through experiments in future.

The particle size for all four types of nanoparticles is setat $50 \mathrm{~nm}$. The analysis is carried out by varying the particle volume fraction between 0.2 to $2 \%$. The thermophysical properties are a function of particle size and temperature as well, therefore, the temperature was varied from 273 to $303 \mathrm{~K}$ for considering the temperature effects and the diameter of nanoparticles was varied from 10 to $70 \mathrm{~nm}$ for considering the particle size effects. All the nanoparticle properties has been summarised in Table 1.

Table I: Thermophysical properties of various nanoparticles[14-18]

\begin{tabular}{ccccc}
\hline & Copper & Aluminium & $\begin{array}{c}\text { Copper } \\
\text { Oxide }\end{array}$ & $\begin{array}{c}\text { Aluminium } \\
\text { Oxide }\end{array}$ \\
$(\mathbf{C u})$ & $(\mathbf{A l})$ & $(\mathbf{C u O})$ & $\left(\mathbf{A l}_{2} \mathbf{O}_{3}\right)$ \\
\hline $\begin{array}{c}\text { Thermal } \\
\text { Conducti-vity } \\
(\text { W/mK) }\end{array}$ & 401 & 237 & 20 & 32 \\
$\begin{array}{c}\text { Specific heat } \\
\text { Capacity }(\mathbf{J} / \mathbf{k g K})\end{array}$ & 385 & 903 & 535.6 & 765 \\
& & & & \\
\hline Density & 8933 & 2702 & 6500 & 3970 \\
$\left(\mathbf{k g} / \mathbf{m}^{\mathbf{3}}\right)$ & & & \\
\hline
\end{tabular}

\subsection{Thermal conductivity investigation}

The thermal conductivity models for nanofluids and nanorefrigerants are mainly based on the several theories proposed for nanofluid conduction. The nanofluid thermal conductivity mechanism and models can be broadly classified into three wide categories as mentioned below:-

1) Static mechanism based models.

2) Dynamic mechanism based models.

3) Combined Static and Dynamic mechanism based models.

The investigation for the thermal conductivity enhancement of nanorefrigerants in this paper is carried out by considering the models based on different classifications mentioned above. The authors believe that the thermal conductivity prediction for nanofluids/nanorefrigerants should not be solely based on static mechanism based models as done by researchers in past. The biggest drawback of any model based on a static mechanism is the assumption of motionless nanoparticles i.e. the nanoparticles are motionless in the suspended solution which may not be necessarily true. The researchers must also consider various dynamic models in their study among which the Brownian motion based models are the most important ones. Therefore, the thermal conductivity enhancement values for each nanorefrigerant are compared by using four different models/correlations based on different mechanisms. The models used for the thermal conductivity prediction are Maxwell Model, Yu-Choi Model, Xuan Model and Hassaniet. al. correlation.

The Maxwell model[20] was the first model used to predict the thermal conductivity of nanofluids and is a typical static mechanism based model. It is important to mention that the Maxwell model was not derived for determining the thermal conductivity; perhaps, it was derived for predicting the electrical conductivity of composite solids as discussed in his work [20]. Carslaw and Jaeger extended the use of Maxwell model for 
determining the thermal conductivity of composite solids arguing that the electrical conductivity and thermal conductivity of solids are two sides of the same coin. Later on, the researchers started utilising the Maxwell model for liquid suspensions such as nanofluids which may not be correct since thermal energy transport mechanism in nanofluids is very different from that of composite solids. This is one of the reasons why experimental values don't match with the predictions made by Maxwell model. The Maxwell model is one of the least accurate models for predicting the thermal conductivity enhancement of nanofluids/nanorefrigerants and is given by:-

$$
k_{n f}=\left[\frac{k_{n p}+2 k_{f}+2 \phi\left(k_{n p}-k_{f}\right)}{k_{n p}+2 k_{f}-\phi\left(k_{n p}-k_{f}\right)}\right] k_{f}
$$

Where $\mathrm{k}_{\mathrm{np}}$ is thermal conductivity of the nanoparticle, $\mathrm{k}_{\mathrm{f}}$ is the thermal conductivity of the base fluid/base refrigerant, $\phi$ is the particle volume fraction and $\mathrm{k}_{\mathrm{nf}}$ is the thermal conductivity of the nanofluid/nanorefrigerant.

Yu-Choi model[21] is another static mechanism based model and is a renovated form of the Maxwell model. The Yu-Choi model is based on the concept of nanolayer. They believed that the nanolayer provides an effective thermal path for heat transfer between the fluid and the nanoparticles which has slightly higher thermal conductivity than the nanoparticle. But, it is difficult to experimentally determine the thermal conductivity of this nanolayer. Therefore, it is advisable to have a conservative approach while assuming the thermal conductivity of the nanolayer .In this work, the thermal conductivity of the nanolayer was assumed to be 5\% higher than the thermal conductivity of nanoparticle.

$$
k_{n f}=\left[\frac{k_{p e}+2 k_{f}+2 \phi\left(k_{n p}-k_{b f}\right)(1+\beta)^{3}}{k_{p e}+2 k_{f}-\phi\left(k_{n p}-k_{b f}\right)(1+\beta)^{3}}\right] k_{f}
$$

Xuan model[22] is given by:-

$$
k_{n f}=\left[\frac{k_{n p}+2 k_{f}+2 \phi\left(k_{n p}-k_{f}\right)}{k_{n p}+2 k_{f}-\phi\left(k_{n p}-k_{f}\right)}\right] k_{b f}+\frac{\rho_{p} \phi c_{p}}{2} \sqrt{\frac{k_{B} T}{3 \pi r_{c l} \mu}}
$$

Where $\mathrm{a}_{\mathrm{cl}}$ is the nanoparticle cluster radius. In this investigation, the nanoparticle cluster radius was assumed to be $100 \mathrm{~nm}$. The particle aggregation leading to high nanoparticle cluster radius pose a threat to the existence of nanofluid itself due to rapid settling of the larger particles. Therefore, the assumption of very high cluster radius is not practical. This is the reason why the cluster radius was assumed to be $100 \mathrm{~nm}$.

The Hassani et al. correlation [23] was developed by using the Vashy-Buckingham theorem based on various experimental data and several factors such as Brownian motion, particle volume fraction, particle size and effects of nanofluid temperature has been incorporated into the correlation. This correlation claims to agree with $98 \%$ of the experimental value within a deviation $+/-10 \%$. The Hassaniet. al. correlation is given by:-

$$
\pi_{1}=1.04+\pi_{2}^{1.11} \pi_{3}^{0.33} \pi_{4}^{-1.7}\left[\frac{1}{\pi_{4}^{-1.7}}-\frac{262}{\pi_{3}^{0.33}}+135 \pi_{5}^{0.23} \pi_{6}^{0.82} \pi_{7}^{-0.1} \pi_{8}^{-7}\right]
$$

where $\pi_{1}=\mathrm{k}_{\mathrm{nf}} / \mathrm{k}_{\mathrm{f}}, \pi_{2}=\phi, \pi_{3}=\mathrm{k}_{\mathrm{np}} / \mathrm{k}_{\mathrm{f}}, \pi_{4}=\operatorname{Pr}, \pi_{5}=\mathrm{d}_{\mathrm{ref}} / \mathrm{d}_{\mathrm{np}}, \pi_{6}=v_{\mathrm{f}} /\left(\mathrm{d}_{\mathrm{np}} \cdot \mathrm{V}_{\mathrm{Br}}\right), \pi_{7}=\mathrm{C}_{\mathrm{p}} /\left(\mathrm{T}^{-1} \cdot \mathrm{V}^{2}{ }_{\mathrm{Br}}\right), \pi_{8}=\mathrm{T}_{\mathrm{b}} / \mathrm{T}$. Here, $\mathrm{d}_{\mathrm{ref}}$ is the reference diameter which is equal to $2.9^{*} 10^{-10}$ and $\mathrm{V}_{\mathrm{br}}$ is the Brownian velocity.

\subsection{Specific Heat and Density Investigation.}

The specific heat and density of nanofluid depend linearly on the particle volume fraction which enables the researchers to predict the values of these two properties much more accurately[28]. The specific heat and density enhancement or reduction values can be predicted on a weight average basis mentioned below:-

$$
\frac{\rho_{n f}}{\rho_{f}}=(1-\phi)+\phi\left(\frac{\rho_{n p}}{\rho_{f}}\right)
$$

Where $\rho n f$ is the density of nanofluid/nanorefrigerant, $\rho f$ is the density of fluid, $\rho n p$ is the density of nanoparticle and $\phi$ is the particle volume fraction.

$$
\frac{c_{p, n f}}{c_{p, f}}=(1-\phi)+\phi\left(\frac{c_{p, n p}}{c_{p, f}}\right)
$$


Where $\mathrm{Cp}, \mathrm{nf}$ is the specific heat capacity of the nanofluid/nanorefrigerant, $\mathrm{Cp}, \mathrm{nf}$ is the specific heat capacity of the nanofluid/nanorefrigerant, $\mathrm{Cp}, \mathrm{nf}$ is the specific heat capacity of the nanoparticles and $\phi$ is the particle volume fraction.

\section{Results and discussion.}

Figs.1-4 show the thermal conductivity enhancement values for various particle volume fraction based on Maxwell model, Yu-Choi model, Xuan model and Hassaniet. al. correlation. It is quite evident from the figures that the Xuan model predicts the highest thermal conductivity enhancement values whereas the Maxwell model predicts the least values for the thermal conductivity enhancement. The Xuan model is combined (static and dynamic) model which incorporates various effects such as the Brownian motion and particle aggregation resulting in a much higher thermal conductivity prediction than the Maxwell model. The Maxwell model, on the other hand, incorporates only the particle volume fraction and the thermal conductivity values of the nanoparticles and the base refrigerant. Hence, the Maxwell model sets a lower limit to the thermal conductivity enhancement values.

Among the four nanorefrigerants under study, the $\mathrm{Cu}-\mathrm{R} 141 \mathrm{~b}$ nanorefrigerant exhibits maximum thermal conductivity enhancement mainly because of extremely high thermal conductivity of the Copper nanoparticles whereas CuO-R141b exhibits the least thermal conductivity enhancement values. The thermal conductivity values for Al-R141b and Al2O3-R141b lie somewhere in between the Cu-R141b and CuO-R141b values. But, when we closely observe the thermal conductivity enhancement plots for these four nanorefrigerants, we would find that there is a big difference between the thermal conductivity values predicted by the Maxwell model and the Xuan model.Therefore, the question arises whether the prediction of the Xuan model is correct or is it the Maxwell model which predicts the correct value. For the right answer, we would have to look into the physics of this problem. Since, the Maxwell model is purely a static mechanism based model which completely ignores the Brownian motion of the particles; clearly under- predicts the thermal conductivity enhancement values of nanorefrigerants and nanofluids. It is evidently clear from this study and from previous experimental results that the Maxwell model sets a lower limit to the thermal conductivity enhancement values. On the contrary, the Xuan model is a combined (static and dynamic) model based on Brownian motion and aggregation of nanoparticles which may not be true for all the nanoparticles in the base fluid/base refrigerant.

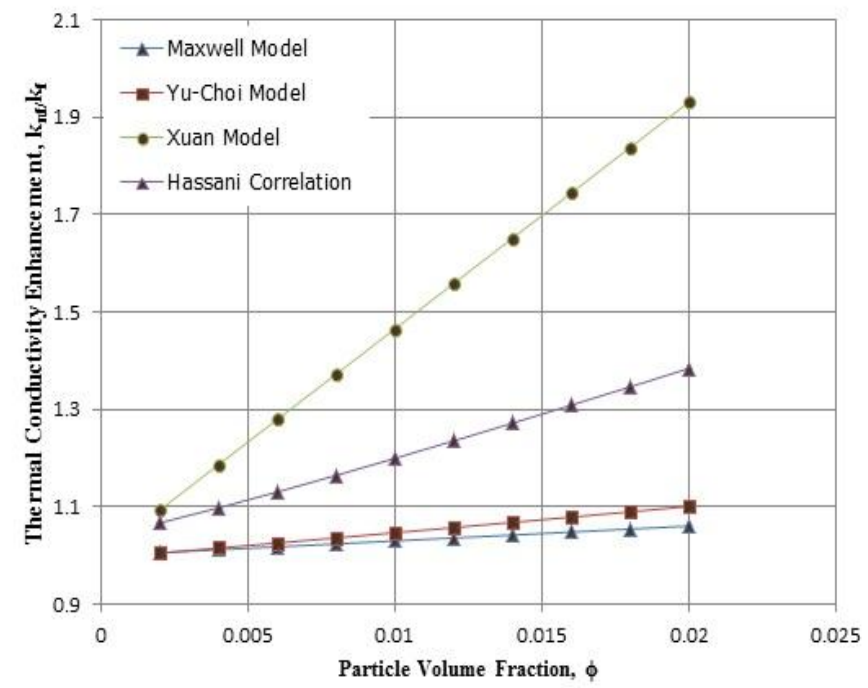

Fig.1Thermal conductivity enhancement prediction of CuO-R141b nanorefrigerant

Therefore, there is a demand for a model/correlation which can predict the thermal conductivity values more accurately and more precisely. The Hassaniet. al. correlation gives more realistic prediction of the thermal 
conductivity enhancement values because a majority of its prediction matches with the previous experimental values within an interval of $+/-10 \%$.

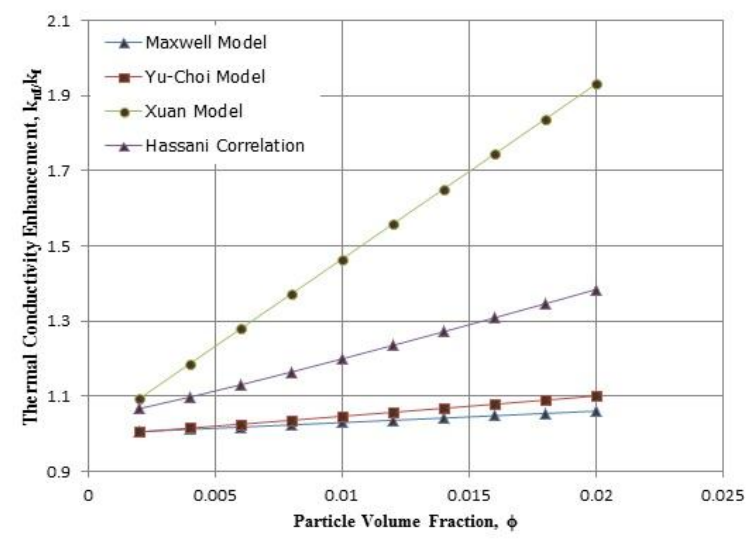

Fig.2: Thermal Conductivity Enhancement Prediction of $\mathrm{Al}_{2} \mathrm{O}_{3}-\mathrm{R} 141 \mathrm{~b}$ nanorefrigerant.

Perhaps, this correlation was developed by using previous experimental data itself which makes it even more reliable. It is important to validate the prediction of these models and correlations by performing experiments but there is a big possibility that the Hassaniet. al. correlation would turn out to be more accurate among these models/correlations. This is the reason why the authors of this work have given maximum importance to the Hassaniet. al. correlation.

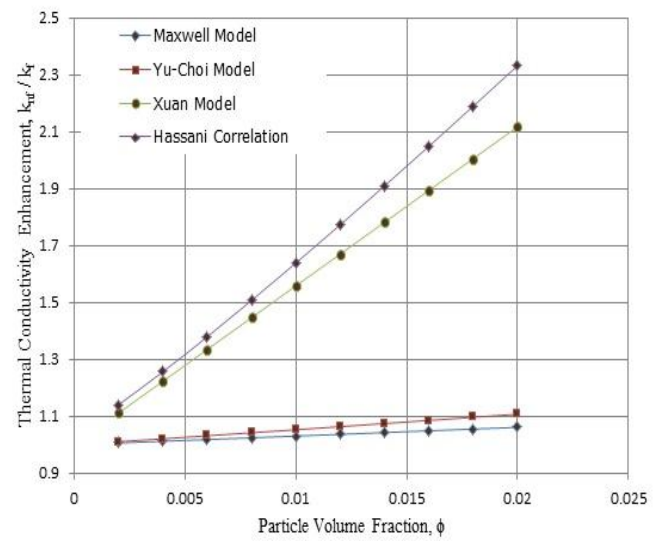

Fig.3: Thermal conductivity enhancement prediction of $\mathrm{Cu}-\mathrm{R} 141 \mathrm{~b}$ nanorefrigerant.

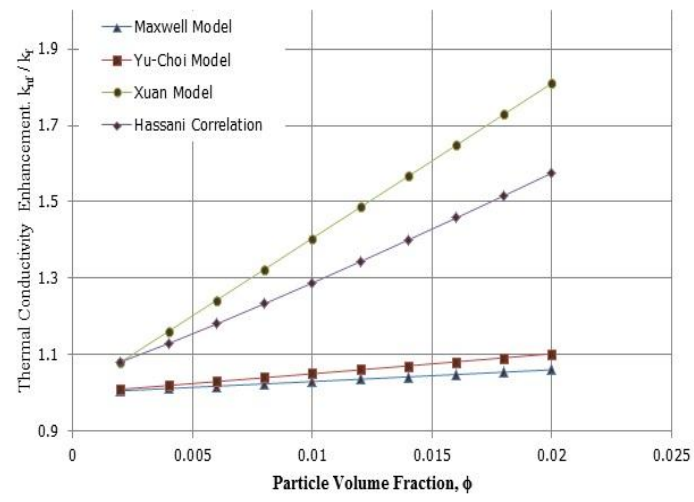

Fig.4: Thermal conductivity enhancement prediction of Al-R141b nanorefrigerant.

Figs 5-6 show the variation in specific heat and density enhancement with varying particle volume fraction. The addition of nanoparticles has an opposing effect on the density and specific heat of the nanorefrigerant. There is an enhancement in the nanorefrigerant density whereas the specific heat capacity reduces with the dispersion of nanoparticles in the base fluid/refrigerant. The $\mathrm{Cu}$ and $\mathrm{CuO}$ based nanorefrigerants exhibit a sharp rise in density enhancement with increasing particle volume fraction whereas the $\mathrm{Al}$ and $\mathrm{Al} 2 \mathrm{O} 3$ based nanorefrigerants exhibit milder rise. The higher density rise has adverse effects on the thermal diffusivity of the nanorefrigerant as discussed later. The specific heat capacity for all nanorefrigerants reduces with the increase in particle volume fraction. $\mathrm{Cu}$ and $\mathrm{CuO}$ based nanorefrigerant undergo highest reduction in the specific heat capacity. 


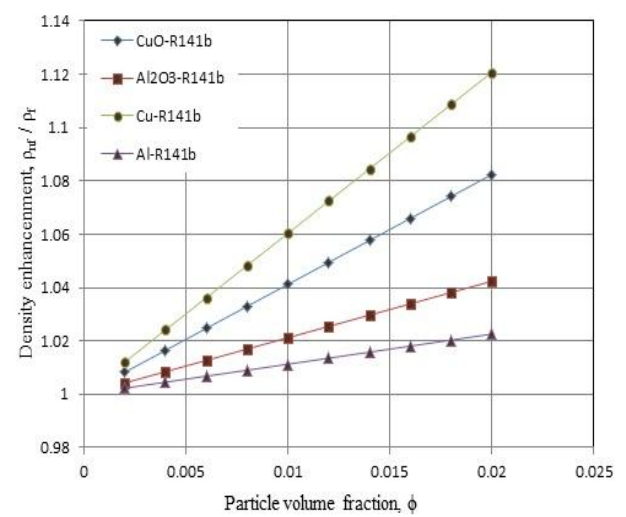

Fig.4: Density enhancement due to the addition of nanoparticles into the refrigerant

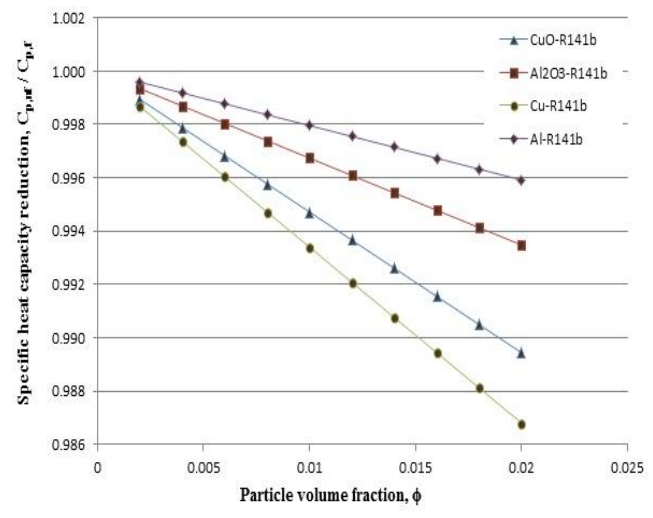

Fig. 6: Specific heat reduction due to the addition of nanoparticles into the refrigerant.

The figures show that there is $2 \%, 4 \%, 8 \%$ and $12 \%$ enhancement in the density of $\mathrm{Al}-\mathrm{R} 141 \mathrm{~b}, \mathrm{Al}_{2} \mathrm{O}_{3}-\mathrm{R} 141 \mathrm{~b}$, $\mathrm{CuO}-\mathrm{R} 141 \mathrm{~b}$ and $\mathrm{Cu}-\mathrm{R} 141 \mathrm{~b}$ respectively at a particle volume fraction of $2 \%$. The specific heat of all the nanorefrigerants reduces by $1-2 \%$ in general.

\section{Conclusions.}

This work studies and compares the thermophysical properties of R141b based nanorefrigerants by utilising various mechanisms and models. The main points to notice in this analysis are mentioned below:-

1) The Maxwell model under-predicts the thermal conductivity enhancement of nanorefrigerants/nanofluids and sets a lower limit to the thermal conductivity enhancement values.

2) The extensive use Maxwell's electrical conductivity equation in the prediction of thermal conductivity of nanofluids doesn't yield accurate results and the theory behind its use for nanofluid thermal conductivity prediction is flawed.

3) Hassani et al. correlation is the conceptually more accurate among all the models/correlation used for thermal conductivity analysis in this work.

\section{References}

[1] IIR. Report on refrigeration sector Achievements and Challenges. The World Summit on Sustainable Development. Johannesburg: 2002.

[2] Bi S, Guo K, Liu Z, Wu J. Performance of a domestic refrigerator using TiO2-R600a nano-refrigerant as working fluid. Energy Convers Manag 2011;52:733-7.

[3] Bi S shan, Shi L, Zhang L li. Application of nanoparticles in domestic refrigerators. Appl Therm.Eng2008;28:1834-43

[4] Nair V, Tailor PR, Parekh AD. Nanorefrigerants:- a comprehensive review on its past, present and future.Int J Refrig 2016;67:290-307.

[5] Cheng L, Liu L. Boiling and two-phase flow phenomena of refrigerant-based nanofluids: Fundamentals, applications and challenges Int J Refrig 2012;36:421-46.

[6] Javadi FS, Saidur R. Energetic, economic and environmental impacts of using nanorefrigerant in domestic refrigerators in Malaysia. Energy Convers Manag 2013;73:335-9.

[7] Henderson K, Park YG, Liu L, Jacobi AM. Flow-boiling heat transfer of R-134a-based nanofluids in a horizontal tube. Int J Heat Mass Transf 2010;53:944-51.

[8] Akhavan-Behabadi MA, Sadoughi MK, Darzi M, Fakoor-Pakdaman M. Experimental study on heat transfer characteristics of R600a/POE/CuO nano-refrigerant flow condensation. ExpTherm Fluid Sci 2015;66:46-52.

[9] Yang D, Sun B, Li H, Fan X. Experimental study on the heat transfer and flow characteristics of nanorefrigerants inside a corrugated tube. Int J Refrig2015;56:213-23.

[10] Tang X, Zhao Y-H, Diao Y. Experimental investigation of the nucleate pool boiling heat transfer characteristics of $\delta$ Al2O3-R141b nanofluids on a horizontal plate. ExpTherm FluidSci2014;52:88-96.

[11] Peng H, Ding G, Hu H, Jiang W. Influence of carbon nanotubes on nucleate pool boiling heat transfer characteristics of 
refrigerant-oil mixture. Int J ThermSci 2010;49:2428-38.

[12] Jiang W, Ding G, Peng H. Measurement and model on thermal conductivities of carbon nanotube nanorefrigerants. Int J ThermSci 2009;48:1108-15.

[13] Sidik NAC, Mohammed HA, Alawi OA, Samion S. A review on preparation methods and challenges of nanofluids. IntCommun Heat Mass Transf 2014;54:115-25.

[14] Touloukian, Y. S. and CYH. Thermophysical Properties of Matter, Vol. 1. New York: Plenum Press; 1972.

[15] Touloukian, Y. S. and CYH. Thermal Conductivity of Metallic Solids; Vol. 2. New York: Plenum Press; 1972.

[16] Touloukian, Y. S. and CYH. Thermal Conductivity of Nonmetallic Solids; Vol. 4. New York: Plenum Press; 1972.

[17] Touloukian, Y. S. and CYH. Specific Heat of Metallic Solids; Vol. 5. New York: Plenum Press; 1972.

[18] Touloukian, Y. S. and CYH. Specific Heat of Nonmetallic Solids; Vol. 7. New York: Plenum Press; 1972.

[19] NIST. Thermphysical properties of fluid systems n.d. http://webbook.nist.gov/chemistry/fluid/.

[20] Maxwell JC. A treatise on electricity and magnetism. Oxford Clarendon Press 1873:360-6. doi:10.1016/00160032(54)90053-8.

[21] Choi SUS. The role of interfacial layers in the enhanced thermal conductivity of nanofluids: A renovated Maxwell model. J Nanoparticle Res 2003; 5:167-71.

[22] Xuan Y, Li Q, Hu W. Aggregation structure and thermal conductivity of nanofluids. AIChE J 2003;49:1038-43. doi:10.1002/aic.690490420.

[23] Hassani S, Saidur R, Mekhilef S, Hepbasli A. A new correlation for predicting the thermal conductivity of nanofluids; using dimensional analysis. Int J Heat Mass Transf 2015;90:121-30. doi:10.1016/j.ijheatmasstransfer.2015.06.040. 